\title{
AVALIAÇÃO DOS RESÍDUOS GERADOS DURANTE A DESTRUIÇÃO DE PCBS PELO PROCESSO CATALISADO POR BASE
}

\author{
Cassio P. Menini ${ }^{\mathrm{a}, \mathrm{b}}$, Kassia S. Kanieski ${ }^{\mathrm{a}}$, Martinho J. Vichinheski ${ }^{\mathrm{a}}$, Tarcisio Loddic, Marco A. Marin ${ }^{\mathrm{d}}$, Marilda Munaro ${ }^{\mathrm{a}}$ e \\ Joseane V. Gulmine ${ }^{\mathrm{a}, *}$

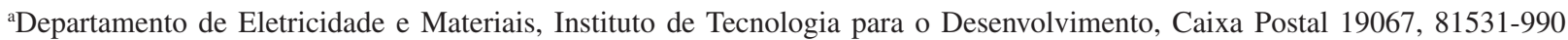 \\ Curitiba - PR, Brasil \\ 'Departamento de Engenharia Mecância, Setor de Tecnologia da Universidade Federal do Paraná, Caixa Postal 19011, 81531-990 \\ Curitiba - PR, Brasil \\ ${ }^{c}$ Copel Distribuição S. A., Rua José Izidoro Biazetto, 158, Bloco C, 81200-240 Curitiba - PR, Brasil \\ ${ }^{\mathrm{d} C o p e l ~ G e r a c ̧ a ̃ o ~ e ~ T r a n s m i s s a ̃ o ~ S . ~ A ., ~ R u a ~ J o s e ́ ~ I z i d o r o ~ B i a z e t t o, ~ 158, ~ B l o c o ~ C, ~ 81200-240, ~ C u r i t i b a ~-~ P R, ~ B r a s i l ~}$
}

Recebido em 17/01/2018; aceito em 28/03/2018; publicado na web em 18/04/2018

\begin{abstract}
EVALUATION OF RESIDUES GENERATED OVER PCBs DESTRCUTION BY BASE CATALYZED PROCESS. There are many processes capable of decontamination of polychlorinated biphenyls in several matrixes. The set of inputs, materials, operation conditions and efficiencies are wide. The base catalyzed dechlorination process shows application potential in great scale, mainly due mild chemical reaction conditions and low cost raw materials. The KPEG is a familiar process of base catalyzed dechlorination. This decontamination treatment was developed mainly on mineral insulating oil. Few information is found related to process residues, usually in reaction sludge means, limited to inform that its destination must fulfill specific territorial laws. In these work KPEG treatment process was performed on decontamination of mineral insulating oils containing between 200 and 330 ppm of polychlorinated biphenyls to evaluation of reaction residues and of the decontamination percentage. Chemical characterization of these residual material allows achievement of important information to explore procedures for recovery, regeneration and/or right disposal. The analysis performed on the residues showed the presence of reaction products containing unsaturation, absents on neat polyethylene glycol, possible organic complexes products with alkali metal as also the presence of chloride ions. In oil was verified the presence of less chlorinated biphenyls, probably formed by partial dechlorination of highly chlorinated biphenyls.
\end{abstract}

Keywords: polychlorinated biphenyls; PCBs; base catalyzed process; decontamination; mineral insulating oil.

\section{INTRODUÇÃO}

Produzidas em larga escala industrial entre as décadas de 1930 e 1960, as bifenilas policloradas (PCBs) são substâncias químicas organocloradas utilizadas como base de óleos sintéticos que foram utilizados para os mais diversos fins industriais. A maior utilização correspondeu ao setor de energia elétrica, como fluido isolante elétrico nos transformadores. No Brasil, estas substâncias foram comercializadas com o nome de Ascarel $^{\circledR}$, sendo compostas por misturas de várias moléculas contendo diferentes quantidades e disposições de átomos de cloro nas bifenilas (congêneres) e clorobenzenos..$^{1-3}$ Devido às características desses óleos, como baixa reatividade química, alta resistência à inflamabilidade e boa rigidez dielétrica, tornaram-se adequados às condições de serviço destes equipamentos elétricos. No entanto, foram observadas doenças crônicas e sintomas de intoxicação associadas à exposição à PCBs, relacionadas com os efeitos carcinogênicos, mutagênicos e bioacumulativos desses produtos quimicos em seres vivos. ${ }^{1-7}$ Esses problemas citados, associados à grande resistência a degradação ambiental, levou a classificação dessas substâncias como poluentes orgânicos persistentes (POPs) pela Convenção de Estocolmo de 2001, sendo que os países signatários, dentre eles o Brasil, estabeleceram o acordo de realizar a total retirada de todas as fontes contaminadas até 2025 e sua total destruição até 2028 , $^{2,3,6}$ No setor elétrico além do montante de PCBs retiradas dos equipamentos atendendo à Convenção, ainda ocorreu a contaminação de óleo mineral isolante (OMI) utilizado para a substituição do Ascarel $^{\circledR}$ nos transformadores de forma inadequada,

*e-mail: jogulmine@yahoo.com.br como, por exemplo, o simples esgotamento do mesmo e enchimento do equipamento com óleo mineral, havendo ainda PCBs impregnadas no papel isolante do transformador. ${ }^{8-10}$

Diante desse cenário, diversos métodos de tratamento para descontaminação de matrizes com PCBs foram criados. ${ }^{11-14}$ Dentre estes, os processos de descloração catalisados por base são tratamentos químicos com menor impacto ambiental que podem gerar a matriz descontaminada (oléo mineral isolante), a lama reacional e gases de exaustão. ${ }^{914,15}$ A lama reacional, geralmente com consistência pastosa, é formada por resíduo da matriz e reagentes não consumidos, além de compostos formados pelo processo de descloração das bifenilas. ${ }^{15,16}$ O processo KPEG, uma das formas de tratamento catalisado por base, recebe esta denominação por utilizar hidróxido de potássio $(\mathrm{KOH})$ e polietilenoglicol (PEG). Este processo é vantajoso, pois é um método simples, seguro, versátil, barato e eficiente comparado aos seus principais concorrentes (incineração e reação com sódio métalico). Alguns trabalhos apresentam hipoteticamente os principais produtos formados durante a reação, baseados em compostos modelo de PCBs, em que o PEG atua tanto como agente de transferência de fase, quanto como doador de hidrogênio para a descloração das bifenilas, produzindo poliaril glicolatos insolúveis em óleo isolante e facilmente separados e removidos, após o resfriamento. ${ }^{3,9,17,18} \mathrm{No}$ entanto, este mecanismo não écomprovado na literatura para misturas comerciais de PCBs e não são apresentados resultados analíticos que provem a formação da ligação química entre as bifenilas e o polietilenoglicol, bem como o mapeamento da descloração, levando em conta as diferenças de reatividade dos congêneres. Segundo a literatura, os resíduos gerados neste processo não apresentariam risco ambiental imediato, desde que dispostos adequadamente por outras 
técnicas como por exemplo incineração. ${ }^{3,9,16,19-24}$ Testes de toxicidade aguda mostraram baixos riscos, mas não foram feitos ainda estudos a médio e longo prazos. ${ }^{9,16}$

A caracterização química dos resíduos gerados na reação pode contribuir no aprofundamento do conhecimento para auxiliar na tomada de decisão com relação à correta destinação desses materiais ou ainda para reutilização em outros segmentos industriais que permitiria geração de valor agregado, redução de passivo ambiental e corte de gastos com destinação. ${ }^{25}$

Neste estudo é apresentada a caracterização química dos resíduos do processo de tratamento KPEG aplicado em óleos minerais isolantes contaminados com misturas comerciais de PCBs, sendo uma mistura de Aroclor $1260^{\circledR}$, que contém majoritariamente congêneres penta, hexa e heptaclorados e outra mistura de Aroclor $1242^{\circledR}$, com congêneres di, tri e tetraclorados, predominantemente. Os resíduos foram analisados por cromatografia gasosa com espectrômetro de massa (GC-MS), espectroscopia vibracional de infravermelho médio com transformada de Fourier acoplado com acessório de reflexão total atenuada (FTIR-ATR), espectroscopia nas regiões ultravioleta e visível (UV-Vis) e espectroscopia com ressonância magnética nuclear (RMN).

\section{PARTE EXPERIMENTAL}

\section{Materiais e métodos}

\section{Materiais}

Para realização dos experimentos foram utilizados os seguintes produtos:

- óleo mineral isolante (OMI) sem uso AV-60 produzido pela Petrobras;

- óleos minerais retirados de transformador contendo altos teores de PCBs dos congêneres de Aroclor $1260^{\circledR}$ e Aroclor $1242^{\circledR}$

- polietilenoglicol (PEG) de massas molares médias 300 e 2000 para síntese (Merck);

- hidróxido de potássio P.A. 85\% (Synth) em escamas;

- gás oxigênio 4.0 analítico (WhiteMartins) e nitrogênio 5.0 ECD (WhiteMartins);

- $\quad$ acetona P.A. 99,5\% (Synth);

- cloreto de amônio P.A. 99,5\% (Vetec);

- n-hexano P.A. 99,9\% (Synth);

- papel filtro quantitativo Unifil C41, faixa preta com diâmetro de $125 \mathrm{~mm}$;

- nove conjuntos de padrões comerciais de PCBs com concentração de $10 \mathrm{mg} \mathrm{L}^{-1}$ de cada congênere em iso-octano da marca AccuStandard ${ }^{\circledR}$ contendo ao todo 209 congêneres;

- cartuchos de extração em fase sólida (SPE) de sulfóxido (Supelclean ${ }^{\mathrm{TM}}$ Sulfoxide, Supelco);

- 1,2,3-triclorobenzeno 99\% (Sigma Adrich)

\section{Preparo das soluções de PCBs}

Para o preparo das soluções de OMI controladamente contaminado com PCBs foi tomada uma alíquota de OMI retirado de transformador contaminado com alto teor de Aroclor $1260^{\circledR}$ e adicionada em OMI novo, obtendo uma solução contendo 218 ppm de PCBs (identificada neste trabalho como solução A). O mesmo procedimento foi feito para o preparo de solução contaminada com Aroclor $1242^{\circledR}$, obtendo-se uma solução com concentração de 322 ppm de congêneres (denominada neste trabalho como solução B). Utilizou-se esta metodologia para garantir soluções contendo a mesma procedência, uma vez que há variações entre lotes de um mesmo tipo de Aroclor $^{\circledR}$ e também para adequar a concentração às faixas de contaminação geralmente encontradas nos óleos minerais dos transformadores contaminados no Brasil. A identificação e quantificação realizadas dos congêneres nas soluções A e B preparadas para os testes foram baseadas na norma CEI IEC 61619. ${ }^{26-28}$

\section{Reações de descontaminação}

Para realização das reações de descontaminação utilizou-se um reator de bancada Mini-reactor 4563 com agitação mecânica por acionamento magnético, com controlador de temperatura e velocidade de rotação Automatic Controller 4848, ambos da marca Parr Instruments. O sistema de filtração a vácuo era composto por bomba (Tecnal), funil e kitassato. Para cada teste no reator, foram colocados $250 \mathrm{~mL}$ de OMI contaminado (solução A ou B) e os reagentes KOH (previamente triturado em liquidificador industrial) e PEG. Foram utilizados $10 \%(\mathrm{~m} / \mathrm{m})$ de PEG em relação ao OMI contaminado e uma proporção molar de 7:1 de KOH em relação ao PEG (mol:mol). Foram utilizados dois PEGs com massas molares médias de 300 e 2000, conforme Tabela 1, para avaliar a influência deste parâmetro na reação. Depois de inseridos os reagentes, o reator era fechado e pressurizado a 20 psi com uma mistura gasosa contendo $96 \%$ de $\mathrm{N}_{2}$ e $4 \%$ de $\mathrm{O}_{2}$. Em seguida, foram iniciados os processos de agitação a $700 \mathrm{rpm}$ e o aquecimento. A contagem do tempo de reação foi iniciada a partir do momento em que a temperatura atingiu o valor pré-estabelecido para o teste ( 100 ou $\left.130{ }^{\circ} \mathrm{C}\right)$. Transcorrido o tempo de reação de cada teste, o sistema foi resfriado naturalmente até a temperatura ambiente, ocorrendo a separação entre as fases pastosa polar $(\mathrm{PEG}+\mathrm{KOH})$ e apolar $(\mathrm{OMI})$. O material foi retirado do reator fazendo-se lavagens com acetona. As fases foram separadas por filtração a vácuo e o filtrado foi aquecido a $60{ }^{\circ} \mathrm{C}$ e lavado com hexano para retirada de resquícios de óleo que tenham permanecido na fase polar. Foi realizada também uma reação controle denominada como RC, contendo os reagentes KOH e PEG de massa molar média 300, mas utilizando OMI sem contaminação com PCB, nas mesmas condições de temperatura, atmosfera, pressão e tempo das reações R1 e R2 (Tabela 1) para avaliação e comparação do resíduo pastoso obtido $(\mathrm{PEG}+\mathrm{KOH})$.

Tabela 1. Condições das reações de descontaminação testadas

\begin{tabular}{lccccc}
\hline Reação & R1 & R2 & R3 & R4 & R5 \\
\hline Solução & A & A & B & B & B \\
PEG & 2000 & 300 & 300 & 300 & 300 \\
Temperatura $\left({ }^{\circ} \mathrm{C}\right)$ & 100 & 100 & 100 & 100 & 130 \\
Tempo $(\mathrm{h})$ & $4 \mathrm{~h}$ & $4 \mathrm{~h}$ & $4 \mathrm{~h}$ & $7 \mathrm{~h}$ & $7 \mathrm{~h}$ \\
\hline
\end{tabular}

\section{Análises de GC/MS}

Para as análises de GC/MS, foi utilizado o cromatógrafo a gás, marca Agilent, modelo 7890 B, acoplado ao espectrômetro de massas, marca Agilent, modelo 5977 A, com amostrador automático e coluna capilar fase HP-5 (5\%-difenil 95\% dimetilpolisiloxano) de dimensões $30 \mathrm{~m} \times 0,25 \mathrm{~mm} \times 0,25 \mu \mathrm{m}$. Foi injetado $1 \mu \mathrm{L}$ de amostra em modo splitless com temperatura do injetor de $225^{\circ} \mathrm{C}$. Foi utilizado hélio como gás de arraste a $1 \mathrm{~mL} \mathrm{~min}{ }^{-1}$. A programação do forno foi feita como segue: temperatura inicial de $60^{\circ} \mathrm{C}$, permanência por 4 minutos, aquecimento a $5{ }^{\circ} \mathrm{C} \min ^{-1}$ até $220^{\circ} \mathrm{C}$, permanência por 5 minutos, aquecimento a $3{ }^{\circ} \mathrm{C} \min ^{-1}$ até $230{ }^{\circ} \mathrm{C}$, permanência por 21 minutos. A temperatura na interface foi mantida a $250{ }^{\circ} \mathrm{C}$. A ionização das moléculas foi realizada por impacto de elétrons. A temperatura da fonte de íons foi de $230{ }^{\circ} \mathrm{C}$ e do quadrupolo de $150{ }^{\circ} \mathrm{C}$. As análises foram realizadas em modo de monitoramento de íons selecionados (SIM - Selected Ion Monitoring). A quantificação foi realizada com base na norma CEI IEC 61619, que considera o fator de resposta de cada analito no cálculo da concentração. ${ }^{26-28}$ Este fator de resposta foi 
calculado com base na biblioteca construída utilizando-se os padrões comerciais dos 209 congêneres de PCBs. Antes da injeção e análise, as amostras passaram por um processo de clean-up em cartucho de SPE, procedimento conforme instruções do fabricante, que tem por objetivo promover a concentração do analito de interesse eliminando as interferências causadas pelo óleo e seus produtos de degradação. Após o pré-tratamento foi adicionado $30 \mu \mathrm{L}$ do padrão interno 1,2,3-triclorobenzeno em cada amostra. $\mathrm{O}$ padrão interno também foi adicionado aos padrões de PCBs utilizados.

\section{Análises de FTIR-ATR}

As análises de espectroscópicas vibracionais de FTIR-ATR foram realizadas em equipamento espectrofotômetro Tensor 27 (fabricante Bruker) com acessório Platinum ATR, com cristal de diamante, utilizando DLATGS (sulfato de triglicina deuterada dopada com L-alanina). As amostras de resíduo foram depositadas diretamente no cristal de diamante, obtendo-se os espectros com 32 varreduras, com resolução de $4 \mathrm{~cm}^{-1}$, na faixa espectral de 4000 a $400 \mathrm{~cm}^{-1}$.

\section{Análises de UV-Vis}

As análises espectroscópicas de UV-Vis foram realizadas no equipamento espectrofotômetro Carry 60 de duplo feixe (fabricante Agilent). Analisaram-se as amostras de resíduo dissolvidas em água destilada ( $\mathrm{R} 1$ com concentração de $0,0021 \mathrm{~g} \mathrm{~mL}^{-1}$, R2 com $0,0002 \mathrm{~g} \mathrm{~mL}^{-1}$ e RC com $0,0025 \mathrm{~g} \mathrm{~mL}^{-1}$ ), com a obtenção dos espectros na faixa de 200 a $800 \mathrm{~nm}$, resolução de $0,5 \mathrm{~nm}$ e cubetas de quartzo com $1 \mathrm{~cm}$ de caminho ótico. A amostra da reação R2 foi mais diluída que as outras duas para adequar sua absortividade espectral.

\section{Análises de ${ }^{13} C$ - $R M N$}

As análises de ${ }^{13} \mathrm{C}$-RMN foram feitas usando tetrametilsilano (TMS) como padrão interno em solução $1 \%$ (v/v) em clorofórmio deuterado, ambos Sigma-Aldrich, P.A., em um equipamento Bruker Advance a $200 \mathrm{MHz}$. As amostras de resíduo foram dissolvidas em clorofórmio deuterado e foi adicionado o padrão de TMS.

\section{RESULTADOS E DISCUSSÃO}

\section{Avaliação dos resíduos encontrados na fase apolar (OMI)}

Para avaliar a descontaminação do óleo, durante o processo, foram feitas análises de GC/MS no OMI contaminado antes e após a reação. Essa quantificação foi realizada para cada congênere individualmente, utilizando seu fator de resposta previamente calculado por meio do uso de padrões. As condições de cada reação e os valores iniciais e após o processo se encontram na Tabela 2. Para as reações conduzidas na solução A (reações R1 e R2), houve significativa redução da quantidade de PCBs após $4 \mathrm{~h}$ de processo, chegandose a valores bem inferiores a $50 \mathrm{ppm}$. Para a solução B (contendo majoritariamente congêneres menos clorados) o teste (reação R3) realizado nas mesmas condições da solução A (solução contendo majoritariamente congêneres mais clorados) não mostrou eficiência no processo de descontaminação, removendo em torno de $56 \%$ dos contaminantes. Por esse motivo foram realizados dois testes aumentando o tempo e a temperatura para tentar melhorar o rendimento da reação. Aumentando somente o tempo de 4 para 7 h (reação R4) não houve melhora na descontaminação, mas aumentando também a temperatura de $100{ }^{\circ} \mathrm{C}$ para $130^{\circ} \mathrm{C}$ (reação R5) se observou significativa diminuição da quantidade de PCBs desta solução.

Os resultados de identificação e quantificação para as reações com OMI contendo congêneres da solução A estão apresentados na Figura 1. Neste trabalho foi utilizada a nomenclatura recomendada na norma IEC 61619 (apresentada no anexo B da norma), que nomeia os congêneres numericamente de PCB1 até PCB209, levando em conta a posição e quantidade de cloros presentes nas bifenilas. Observa-se que na amostra inicial a grande maioria dos congêneres presentes eram penta, hexa e heptaclorados. Após a reação estes foram praticamente eliminados do óleo, restando principalmente os tetraclorados, alguns tri, di e monoclorados. Nota-se aumento da concentração das PCBs 1, 18 e 31 em ambas as reações (R1 e R2), provavelmente devido à descloração parcial de congêneres mais clorados. A massa molar média do polietilenoglicol utilizado (300 ou 2000) teve pouca influência no resultado final da descontaminação destes testes (vide valores finais de concentração após as reações, apresentados na Tabela 2), havendo pequena diferença na reatividade dos congêneres eliminados e/ou formados.

Na Figura 2 estão apresentados os resultados para os testes de descontaminação da solução B, com maior tempo e temperatura de reação. Observa-se que, majoritariamente, esta amostra continha congêneres di, tri e tetraclorados antes da reação. Na reação R4 com tempo de $7 \mathrm{~h}$ e temperatura de $100^{\circ} \mathrm{C}$ houve a eliminação dos penta e hexaclorados e a redução dos menos clorados que atingiu valor final de 142,9 ppm. Com a reação sendo conduzida a $130{ }^{\circ} \mathrm{C}$ (reação R5 da Tabela 2) a descontaminação foi mais eficiente, reduzindo significativamente a quantidade dos congêneres presentes no início, eliminando além dos penta e hexaclorados, também todos os tetra e praticamente todos os triclorados. Nesta reação restaram somente os di e monoclorados, além de pequena quantidade da PCB18 triclorada. Houve a formação e aumento de congêneres monoclorados, pela possível descloração parcial dos mais clorados, chegando à quantidade total de 11,3 ppm após o processo.

Como apontado por Mantzavinos et al., o início da formação de radicais livres no PEG começa pela abstração de um radical hidrogênio $(\mathrm{H} \bullet)$ da cadeia principal devido a cisão homolítica causada por temperatura, presença de oxigênio ou ambos. ${ }^{29} \mathrm{Um}$ radical hidrogênio é altamente reativo e sua interação com PCBs fornece excelente potencial para promover a hidrodescloração. No entanto, outras formas de hidrodescloração a partir de doador de hidrogênio, como é o caso do PEG, que pode fornecer os hidrogênios dos grupos $-\mathrm{OH}$ das extremidades de cadeia, geralmente estão apoiadas na formação de íon hidreto, um forte agente nucleófilo capaz de realizar a remoção de átomos de cloro da estrutura da bifenila. ${ }^{22}$ Uma hipótese para explicar o alto teor de descontaminação nos testes realizados neste trabalho poderia ser a descloração total de grande número de PCBs, formando bifenilas e/ou a quebra das ligações entre as fenilas formando clorobenzenos e benzeno. Para averiguar isto foi realizada a análise de GC/MS pesquisando a presença dessas substâncias no óleo de uma das reações que obteve bom desempenho de remoção de PCBs (R2), mas o detector de massas não identificou qualquer destes compostos. Com este resultado, somente o mecanismo de hidrodescloração não explicaria a descontaminação observada, sendo forte indício de que outro mecanismo também estaria ocorrendo concomitantemente, transformando e/ou transferindo boa parte das PCBs para a fase de polietilenoglicol.

Tabela 2. Resultados das quantificação das PCBs ao final do processo de descontaminação

\begin{tabular}{lccccccccccc}
\hline Reação & \multicolumn{2}{c}{ R1 } & \multicolumn{2}{c}{ R2 } & \multicolumn{2}{c}{ R3 } & \multicolumn{2}{c}{ R4 } & \multicolumn{2}{c}{ R5 } \\
\hline \multirow{2}{*}{ Concentração (ppm) } & inicial & final & inicial & final & inicial & final & inicial & final & inicial & final \\
& 218,3 & 9,7 & 218,3 & 11,0 & 322,6 & 142,7 & 322,6 & 142,9 & 322,6 & 11,3 \\
\hline
\end{tabular}



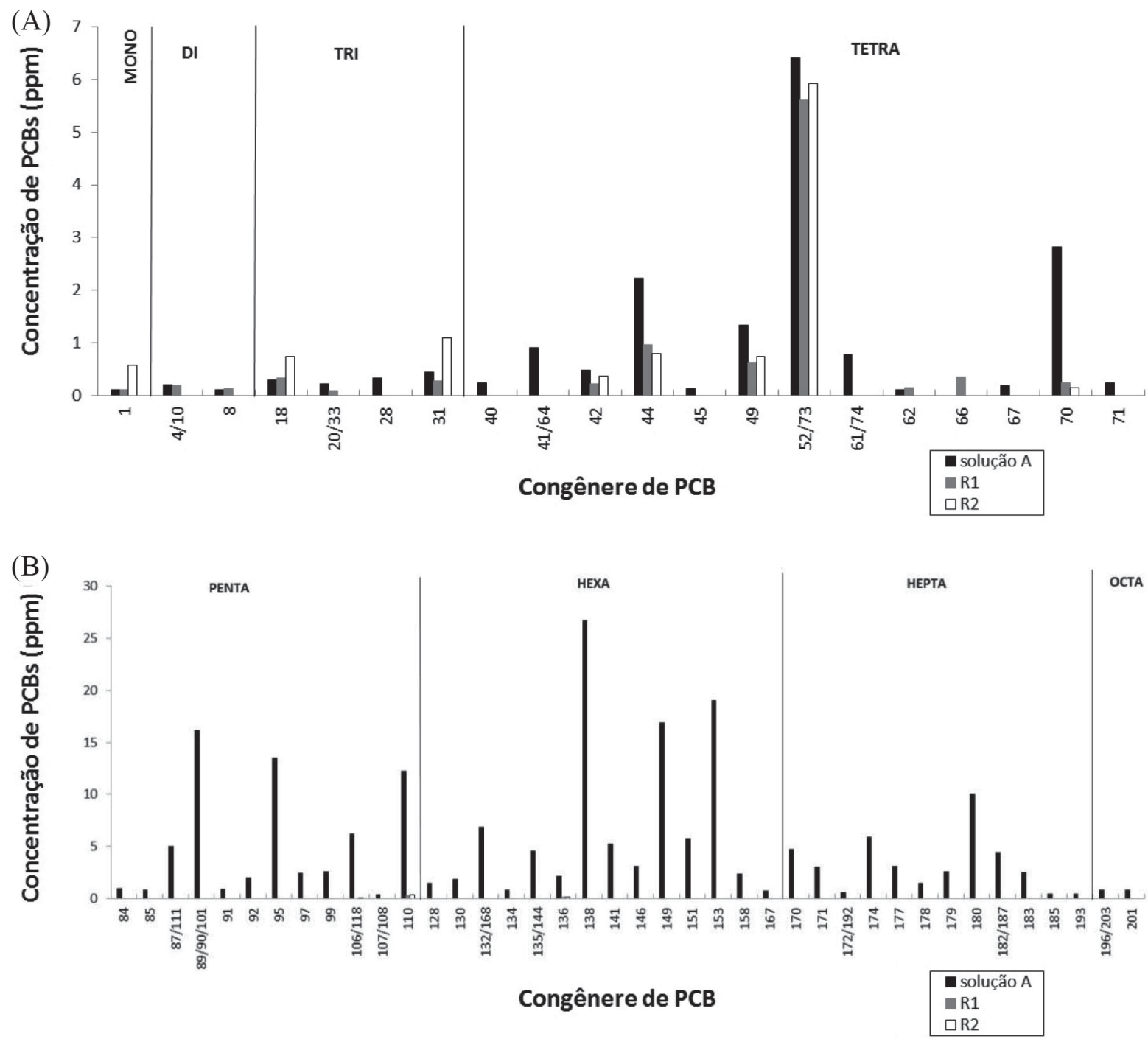

Figura 1. Quantificação dos congêneres de PCBs antes e após a reação de descontaminação, utilizando a solução A e diferentes massas molares do PEG - R1 utilizando PEG 2000 e R2 utilizando PEG 300: A) congêneres mono, di, tri e tetraclorados e B) congêneres penta, hexa, hepta e octaclorados

\section{Avaliação dos resíduos encontrados na fase polar (PEG)}

Uma alternativa que explicaria a eficiência de descontaminação do óleo observada seria a reação entre o PEG e as PCBs, ocorrendo a ligação química entre o oxigênio do grupo álcool na ponta de cadeia do PEG com as PCBs, reação essa que já foi observada em substâncias aromáticas organocloradas e álcoois primários. ${ }^{30}$ Para avaliar essa hipótese foram realizadas análises espectroscópicas de FTIR-ATR, UV-Vis e ${ }^{13} \mathrm{C}-\mathrm{RMN}$ no resíduo pastoso da fase polar.

Na Figura 3 estão apresentados os resultados de espectroscopia vibracional de infravermelho, nos quais se observam bandas referentes à estrutura química do PEG300. As bandas com maiores absorbâncias nos intervalos de 3540 a $3200 \mathrm{~cm}^{-1}, 3000$ a $2840 \mathrm{~cm}^{-1}$ e 1150 a $1085 \mathrm{~cm}^{-1}$, correspondentes às ligações oxigênio-hidrogênio $(\mathrm{O}-\mathrm{H})$, carbono-hidrogênio $(\mathrm{C}-\mathrm{H})$ e carbono-oxigênio-carbono (C-O-C), respectivamente. Nos resíduos das reações, inclusive da reação controle RC, observa-se o aparecimento de uma banda em torno de $1645 \mathrm{~cm}^{-1}$. Essa região é referente a ligações duplas entre carbonos (-C=C-), que podem ter sido geradas pela degradação do PEG no decorrer da reação, segundo mecanismo apresentado na literatura. ${ }^{29}$ Vale esclarecer que poderia ocorrer uma pequeníssima contribuição de ligações duplas dos anéis aromáticos presentes na composição do OMI e de seu antioxidante nesta banda, mas pela quantidades relativas dessas substâncias presentes no óleo e ainda pela pequena porção de óleo que tenha ficado impregnado na fase polar, a concentração provavelmente estaria abaixo do limite de detecção da técnica analítica, não influenciando significativamente a intensidade desta banda. Não foi possível observar bandas referentes à ligação entre $\mathrm{C}-\mathrm{Cl}$ que comprovariam a presença das $\mathrm{PCBs}$ nos resíduos sólidos do PEG e nem ligações entre anel aromático e oxigênio que corroborassem com as propostas de mecanismo da literatura que defendem a formação de ligação química entre algum carbono do anel das bifenilas e o oxigênio presente na ponta da cadeia do PEG (no grupo -OH terminal), talvez pelas baixas concentrações desses grupos químicos que poderiam ser formados, ficando abaixo do limite de detecção do FTIR-ATR.

Na Figura 4 estão apresentados os espectros de UV-Vis do PEG300, do resíduo da reação controle (RC) e do óleo mineral isolante (OMI) com e sem PCBs, além dos espectros das reações de descontaminação dos óleos da solução A. Observa-se que o PEG300 não possui absorção acima de $250 \mathrm{~nm}$, o que era esperado visto que este material quando novo não contém duplas ligações em suas moléculas. O óleo mineral, por sua vez, apresentou uma banda em torno de $270 \mathrm{~nm}$ que deve ser proveniente dos compostos aromáticos que fazem parte de sua composição (em torno de $4 \%$ ), além da contribuição de seu aditivo antioxidante 2,6-di-terc-butil 

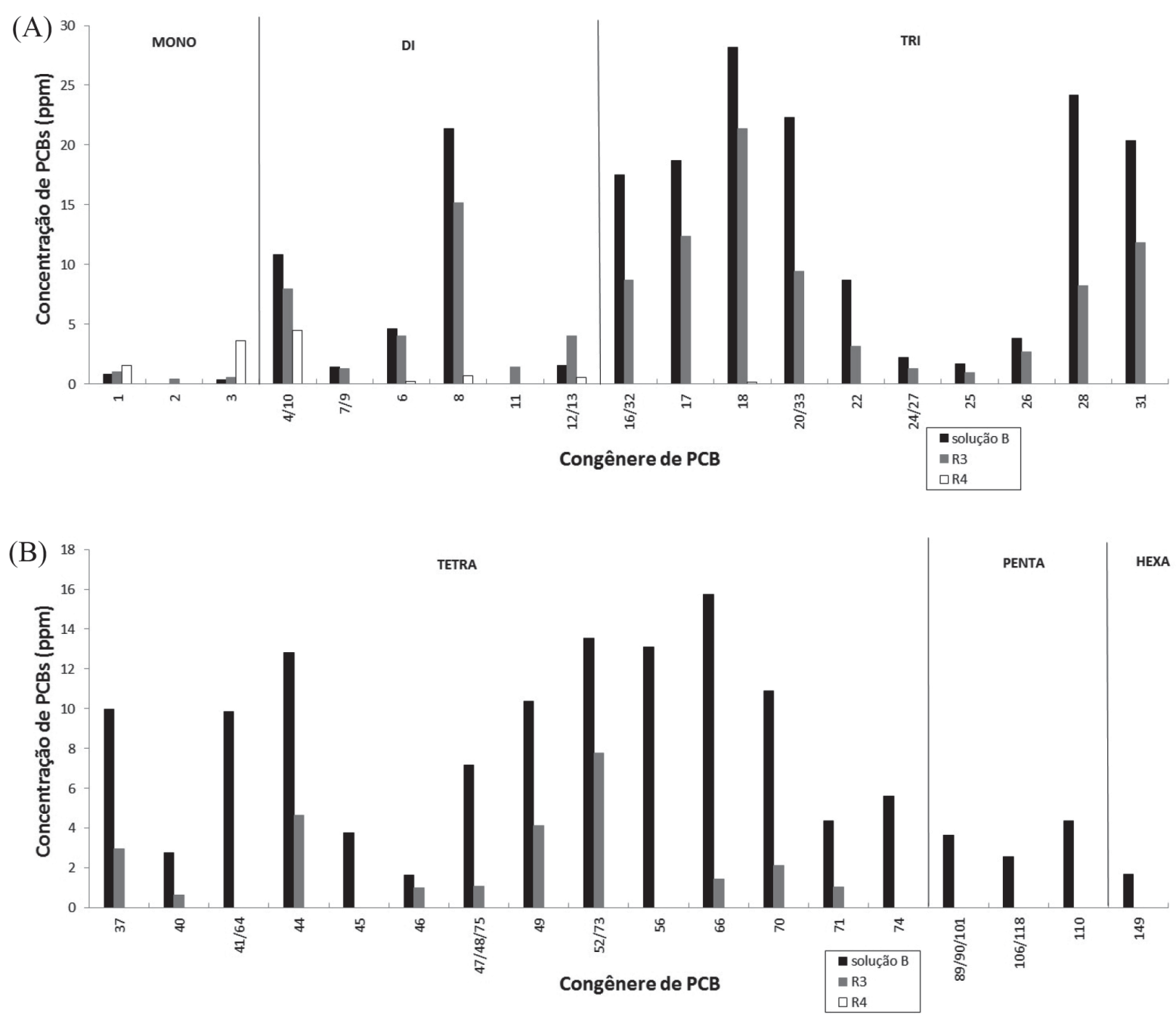

Figura 2. Quantificação dos congêneres de PCBs antes e após a reação de descontaminação, utilizando a solução B e diferentes temperaturas de reação - R4 a $100{ }^{\circ} \mathrm{C}$ e $\mathrm{R} 5$ a $130^{\circ} \mathrm{C}$ : A) congêneres mono, di, e triclorados e B) congêneres tetra, penta e hexaclorados

p-cresol (DBPC), que apresenta também um anel aromático em sua molécula. Foi feita a análise também do óleo mineral contendo PCBs (solução A), não havendo diferença entre os espectros deste óleo e do óleo não contaminado. Isto pode ser devido à baixa concentração de PCBs presentes no óleo contaminado (218,3 ppm na solução A analisada), podendo estar abaixo do limite de detecção da técnica, pois, segundo Andersson et. al., as PCBs apresentariam bandas em 200 a $225 \mathrm{~nm}$ e 245 a $265 \mathrm{~nm}^{31}$

$\mathrm{O}$ resíduo da reação $\mathrm{RC}$ e das reações de descontaminação apresentaram bandas em 270 e $353 \mathrm{~nm}$. A primeira banda pode estar relacionada com a presença de resquícios de óleo mineral impregnado no resíduo polar de PEG e KOH. A atribuição para a segunda banda, em $353 \mathrm{~nm}$, pode estar relacionada com a formação de um complexo entre o PEG e os cátions do potássio provenientes no $\mathrm{KOH}$. A hipótese da formação deste complexo é defendida no trabalho de Brunelle. ${ }^{15}$ Também segundo outros trabalhos na literatura envolvendo a síntese e caracterização de complexos organometálicos, estes compostos absorvem em comprimentos de onda acima $300 \mathrm{~nm} .{ }^{32-34}$

Com o intuito de investigar a formação de ligação química entre as PCBs e o PEG, foi realizada também a análise dos resíduos das reações da solução A por ${ }^{13} \mathrm{C}-\mathrm{RMN}$. O espectro do resíduo R1 que utilizou PEG2000 (Figura 5) mostra picos intensos de deslocamento químico em 70-72 ppm referentes a carbonos da estrutura das cadeias poliméricas do PEG. Foi observado também um pico em 87 ppm que provavelmente está relacionado a carbonos de ligações duplas e/ou triplas. Este pico poderia também estar relacionado com carbonos ligados ao grupo hidroxila de álcool e/ou oxigênio de éter, mas neste caso seria esperado que estivesse presente também no espectro do resíduo RC, o que não ocorreu. O pico em 152 ppm tem relação com carbonos de alcenos, mas também com heteroaromáticos. Muito provavelmente este pico tenha relação somente com insaturações criadas pela degradação do PEG, pois, segundo Egolf, as bifenilas policloradas apresentariam pico em torno de $130 \mathrm{ppm}$ de deslocamento químico. ${ }^{35} \mathrm{O}$ pico em 76 ppm é referente ao solvente clorofórmio deuterado.

Na Figura 6 é mostrado o espectro de ${ }^{13} \mathrm{C}-\mathrm{RMN}$, obtido para o resíduo da reação R2, que foi realizada com PEG 300. Observa-se que os picos mais intensos são praticamente os mesmos encontrados no espectro do PEG 2000 (Figura 5). O pico intenso em 76 ppm é referente ao solvente clorofórmio deuterado.

Os resultados obtidos com a análise dos óleos após as reações mostram que, provavelmente, vários congêneres migraram para a fase polar de polietilenoglicol, mas pelas técnicas analíticas aplicadas aos resíduos pastosos não foi possível saber se elas estão somente aderidas ao resíduo ou quimicamente ligadas ao PEG, muito provavelmente pela quantidade de PCBs estar abaixo do limite de detecção das técnicas espectroscópicas utilizadas. Para investigar este aspecto, foi realizada a análise por GC/MS do solvente hexano utilizado para lavagem do resíduo de PEG da reação R2, com o intuito de investigar a presença de PCBs na fase polar. Caso as bifenilas policloradas 
estivessem apenas adsorvidas no resíduo, estas seriam extraídas passando para o solvente e seriam detectadas no espectrômetro de massas. Após a análise foram encontrados apenas 11 congêneres,
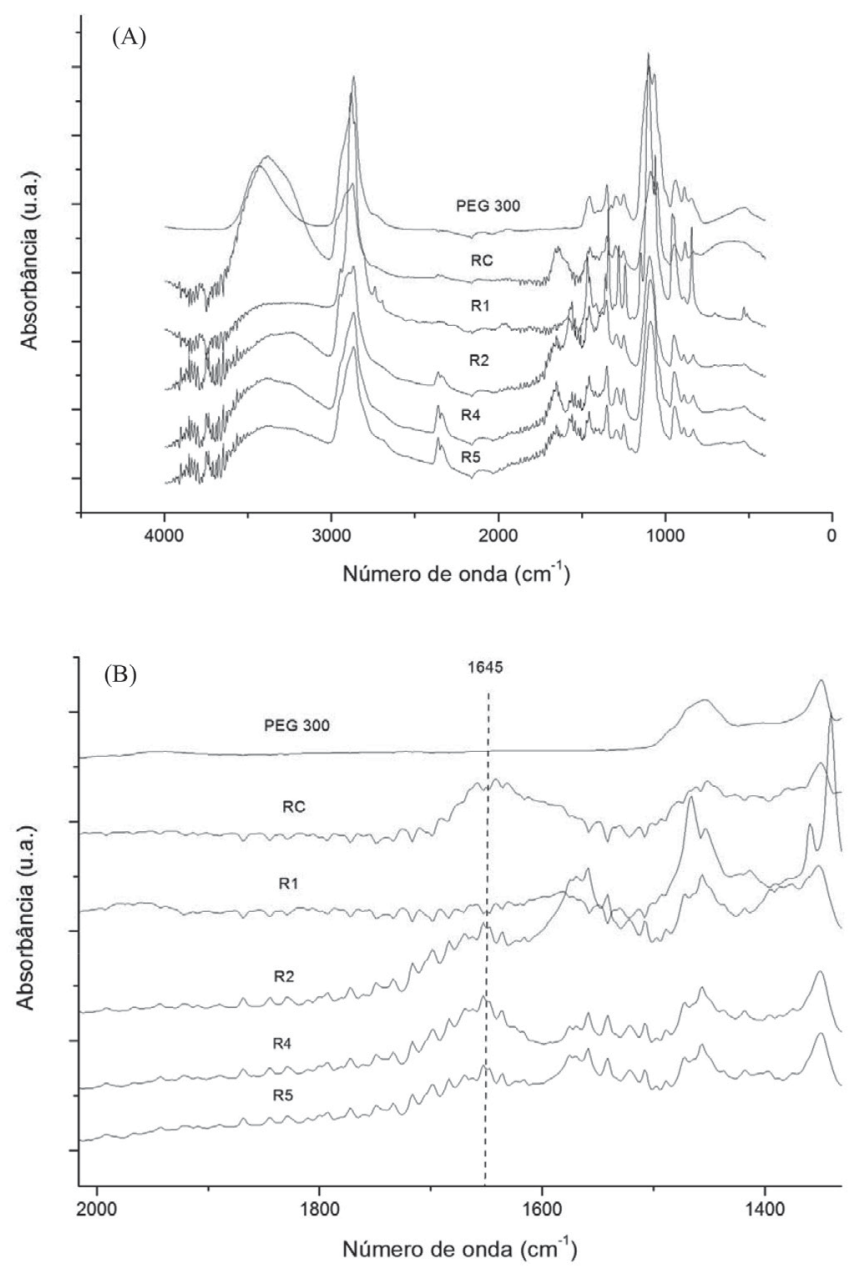

Figura 3. Espectros FTIR-ATR do PEG300, resíduo RC e resíduos das reações de descontaminação: A) região espectral analisada e B) ampliação entre 2000 e $1300 \mathrm{~cm}^{-1}$

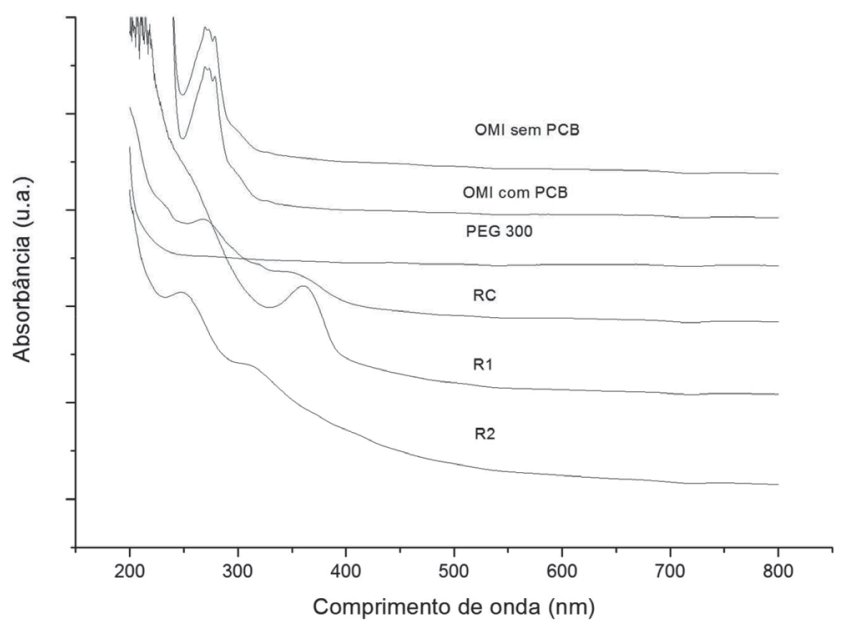

Figura 4. Espectros UV-Vis do óleo mineral isolante, PEG300, resíduo RC e resíduos R1e $R 2$

os mesmos encontrados na análise do óleo após a reação R2 de descontaminação (ver Figura 1), sendo que inicialmente estavam presentes 75 congêneres. Este resultado pode indicar que os 64 congêneres que foram extraídos do óleo estejam ligados quimicamente ao PEG, formando um novo material polimérico, embora não tenha sido possível identificar em qual região da cadeia polimérica do PEG esta ligação se forma.

\section{CONCLUSÃO}

Todos os tratamentos realizados apresentaram percentuais de descontaminação do óleo. Pelos resultados obtidos, dois processos de descloração estão ocorrendo durante as reações. Um deles, que levou ao aumento de alguns congêneres menos clorados inicialmente existentes ou formação de novos, identificados nos óleos, devido a um processo de hidrodescloração, em que átomos de cloro são retirados da molécula de PCB e substituídos por átomos de hidrogênio. $\mathrm{O}$ outro processo leva à abstração de cloro da $\mathrm{PCB}$, com a formação de uma ligação química entre o carbono da bifenila da qual foi retirado o cloro e a cadeia polimérica do PEG, formando um novo material polimérico.

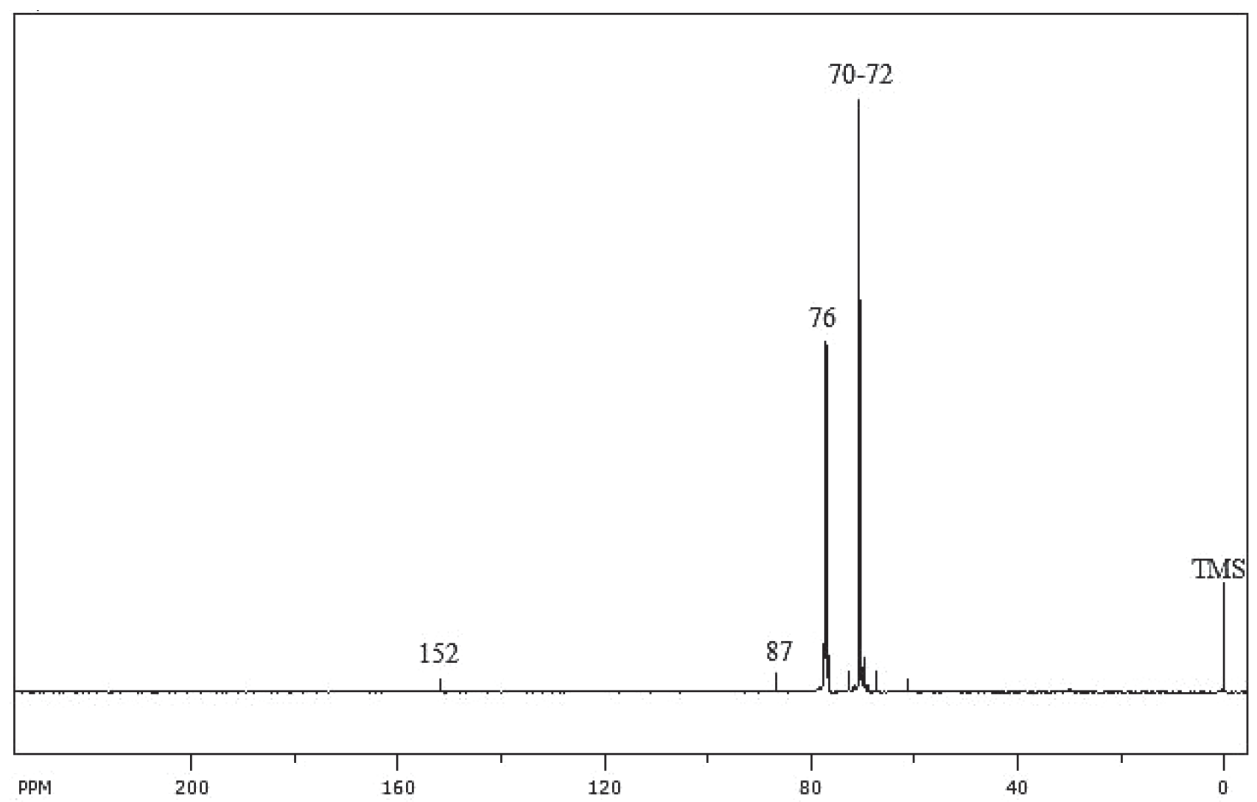

Figura 5. Espectro de ${ }^{13} \mathrm{C}-\mathrm{RMN}$ do resíduo da reação teste $R I$ 


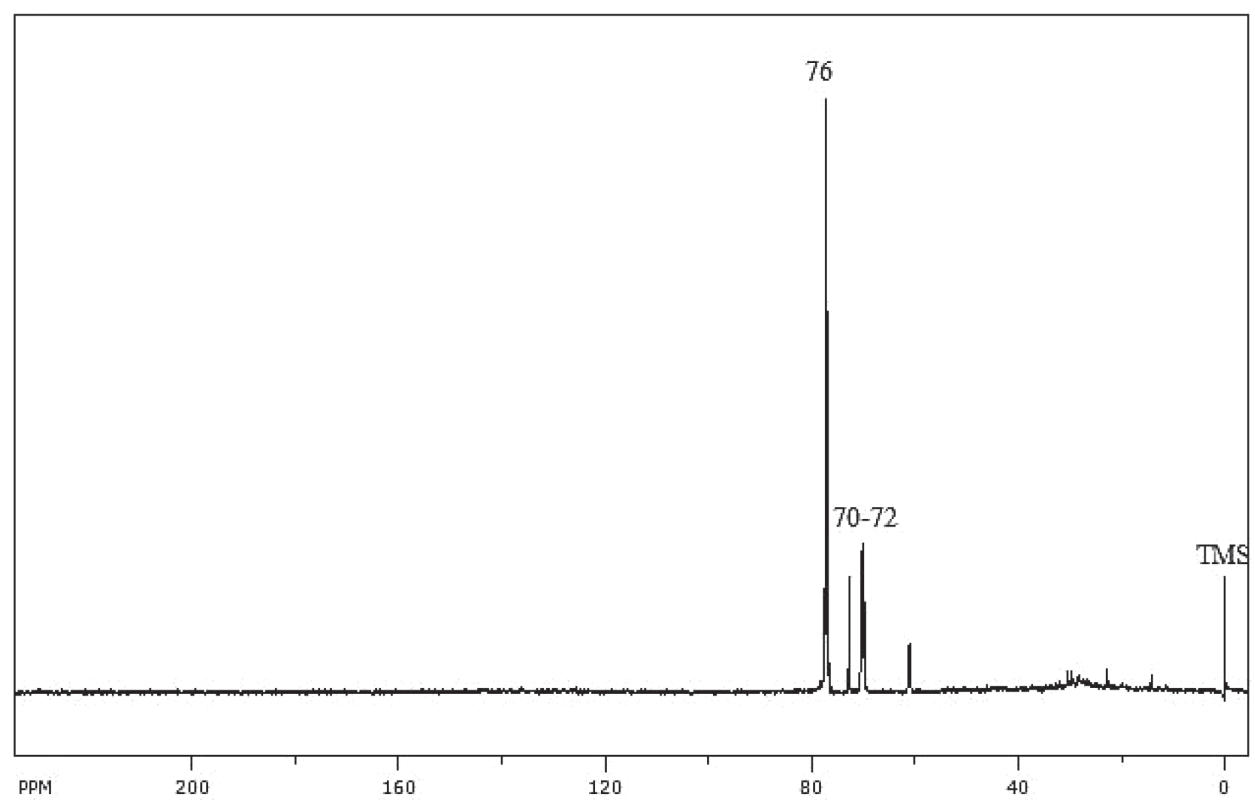

Figura 6. Espectro de ${ }^{13} \mathrm{C}-\mathrm{RMN}$ do resíduo da reação teste $R 2$

A massa molar do PEG parece não influenciar em grande extensão nos teores de descontaminação do óleo, bem como nos resíduos formados que contem fragmentos de cadeia e formação duplas ligações $\mathrm{C}=\mathrm{C}$, evidenciadas por FTIR-ATR, UV-Vis e ${ }^{13} \mathrm{C}-\mathrm{RMN}$.

\section{AGRADECIMENTOS}

Os autores agradecem à ANEEL pelo Programa de $\mathrm{P} \& \mathrm{D}$, à COPEL DIS e à COPEL GET pelo suporte financeiro, ao CNPq pelo benefício da Lei 8010/90, à CAPES, à UFPR e aos Institutos Lactec.

\section{REFERÊNCIAS}

1. Qi, Z.; Buekens, A.; Liu, J.; Chen, T.; Lu, S.; Li, X.; Cen, K.; Environ. Sci. Pollut. Res. 2014, 21, 6448.

2. Wua, B.; Chen, H.; Wang, S. J.; Wai, C. M.; Liao, W.; Chiu, K.; Chemosphere 2012, 88, 757.

3. Gorbunova, T. I.; Saloutin, V. I.; Chupakhin, O. N.; Russ. Chem. Rev. 2010, 79, 511.

4. Tanabe, S.; Environ. Pollut. 1988, 50, 5.

5. Penteado, J. C. P.; Vaz, J. M.; Quim. Nova 2001, 24, 390.

6. Almeida, F. V.; Centeno, A. J.; Quim. Nova 2007, 30, 1976.

7. Jardim, A. N. O.; Caldas, E. D.; Quim. Nova 2009, 32, 1898.

8. Ryoo, K.; Byun, S.; Choi, J.; Hong, Y.; Ryu, Y.; Song, J.; Lee, D.; Lee, H.; Bull. Korean Chem. Soc. 2007, 28, 520.

9. Brunelle, D. J.; Mendiratta, A. K.; Singleton, D. A.; Environ. Sci. Technol. 1985, 19, 740.

10. Cassifi, A.; Beduschi, S.; Balacco, V.; Sacchi, B.; Trasatti, S. P.; Environ. Chem. Lett. 2007, 5, 101.

11. Carpenter, B. H.; Wilson, D. L.; J. Hazard. Mater. 1988, 17, 125.

12. Gomes, H. I.; Dias-Ferreira, C.; Ribeiro, A. B.; Sci. Total Environ. 2013, 445, 237.

13. Monguchi, Y.; Ishihara, S.; Ido, A.; Niikawa, M.; Kamiya, K; Sawama, Y.; Nagase, H.; Sajik, H.; Org. Process Res. Dev. 2010, 14, 1140.

14. Hu, X.; Zhu, J.; Ding, Q.; J. Hazard. Mater. 2011, 191, 258.

15. Noma, Y.; Mitsuhara, Y.; Matsuyama, K.; Sakai, S.; Chemosphere 2007, $68,871$.

16. Janis, W. J. Ferrie, J. S. Braun, J. M.; IEEE Trans. Power Appar. Syst. 1983, 102, 3928.
17. Brunelle, D. J; Singleton, D. A.; Chemosphere 1983, 12, 183.

18. Rahuman, M.S.M.M.; Pistone, L.; Trifirò, F.; Mietus, S.; Destruction Technologies for polychlorinated biphenyls ( $P C B$ 's), International Centre for Science and High Technology United Nations Industrial Development Organization (ICS-UNIDO), Trieste, 2000.

19. Kamarehie, B.; Jafari, A. J.; Mahabadi, H. A.; J. Mater. Cycles Waste Manage. 2014, 16, 711.

20. Kamarehie, B.; Mahabadi, H. A.; Jafari, A. J.; Iran. J. Toxicol. 2014, 8, 1114.

21. Kastanek, P.; Kastanek, F.; Hajek, M.; Sobek, J.; Solcova, O.; Global NEST J. 2011, 13, 59.

22. Ukisu, Y.; Miyadera, T.; Appl. Catal., A 2004, 271, 165.

23. Vijgen, J.; McDowall, R.; Updated POPs Technology Specification and Data Sheets, IHPA, 2009.

24. Egorova, D. O.; Gorbanova, T. I.; Pervova, M. G.; Demakov, V. A.; Appl. Biochem. Microbiol. 2014, 50, 722.

25. Gorbunova, T. I.; Pervova, M. G.; Trushina, E. B.; Pavlyshko, S. V.; Zapevalov, A. Y.; Saloutin, V. I.; Russ. J. Appl. Chem. 2012, 85, 1622.

26. IEC; IEC 61619. Insulating liquids - Contamination by polychlorinated biphenyls (PCBs) - Methods of determination by capillary column gas chromatography, 1997.

27. Santos, K.; Motta, H. N.; Campos, E. A.; Gulmine, J. V.; Munaro, M.; Quim. Nova 2015, 38, 471.

28. Antonello, I.; Humeres, E.; Souza, I. G.; Debacher, N. A.; Quim. Nova 2007, 30, 709 .

29. Mantzavinos, D.; Hellenbrand, R.; Livingston, A. G.; Metcalfe, I. S.; Appl. Catal., B 1996, 11, 99.

30. Shabany, H.; Abel, E.; Evans, J. P.; McRobie, I. M.; Gokel, G. W.; Tetrahedron Lett. 2000, 41, 6705.

31. Andersson, P. L.; Haglund, P.; Tysklind, M.; Fresenius' J. Anal. Chem. 1997, 357, 1088.

32. Pal, A. K.; Laramée-Milette, B.; Hanan, G. S.; Inorg. Chim. Acta 2014, $418,15$.

33. Zhang, Z.; Wu, X.; Sun, F.; Shan, F.; Chen, J.; Chen, L.; Zhou, Y.; Mei, W.; Inorg. Chim. Acta 2014, 418, 23.

34. Kalinowska, M.; Piekut, J.; Bruss, A.; Follet, C.; Sienkiewicz-Gromiuk, J.; Swisłocka, R.; Rzaczynska, Z.; Lewandowski, W.; Spectrochim. Acta, Part A 2014, 122, 631.

35. Egolf, D. S.; Jurs, P. C. Anal. Chem. 1990, 62, 1746. 